\title{
Standard Model Higgs Boson Searches at CDF
}

\author{
Michelle Stancari, on behalf of the CDF collaboration ${ }^{\mathrm{a}}$ \\ ${ }^{a}$ Fermi National Accelerator Laboratory, Batavia, Illinois 60510, USA
}

\begin{abstract}
We present recent results from searches for a standard model Higgs boson by the CDF experiment at the Tevatron $p \bar{p}$ collider with the full Run II data set. An excess of events above the expected background is observed and is the strongest in the associated production search channels where the Higgs is produced together with a $\mathrm{W}$ or $\mathrm{Z}$ boson, and then decays to a bottom-antibottom quark pair, with a global significance of $2.5 \sigma$. Both limits and best fit values of the Higgs production cross section are presented. For a Higgs mass of $125 \mathrm{GeV} / \mathrm{c}^{2}$, the best agreement with data in the $\left(\sigma_{W H}+\sigma_{Z H}\right) \times B r(H \rightarrow b \bar{b})=291 \pm_{113}^{118} \mathrm{fb}$.
\end{abstract}

Keywords: Higgs boson, Tevatron, standard model

\section{Introduction}

In the standard model (SM) of particle physics, electroweak symmetry breaking generates a fundamental scalar boson known as the Higgs boson. However, the standard model does not predict its mass. Only after decades of searching with increasingly more powerful tools has experimental access to the Higgs boson become possible thanks to the large data sets collected by the Tevatron at $\sqrt{s}=2 \mathrm{TeV}$ and the Large Hadron Collider (LHC) at $\sqrt{s}=7$ and $8 \mathrm{TeV}$.

The higher energy of the LHC collisions results in a higher statistical power for the same integrated luminosity for most of the Higgs search channels. However, the Tevatron Run-II data set has a comparable sensitivity to the recent LHC results for the associated production channel $q \bar{q} \rightarrow V H \rightarrow l l b \bar{b}$, where the Higgs decays to a bottom-antibottom quark pair and the vector boson to a lepton pair [1][2], due to the larger ratio of signal to background production cross sections at the Tevatron for this channel.

\section{CDF detector}

The CDF detector [3] is a general purpose detector with cylindrical geometry. The layout of the inner tracking detectors is shown in figure 1. The momentum of charged particles is measured with silicon detectors and an open-cell drift chamber (COT) inside the 1.4T field of a superconducting solenoid magnet. The silicon vertex detector (SVX) identifies the displaced vertices characteristic of long-lived bottom and charm hadron decays both for online trigger event selection and offline analyses. Calorimeters and muon systems located outside the solenoid measure the energy of jets and electromagnetic particles and identify leptons.

\section{Higgs Boson Search Strategies}

The standard model Higgs boson production cross sections at the Tevatron are shown in figure 2 as a function of the mass of the Higgs and the decay branching ratios are shown in figure 3 [4]. For a mass below $130 \mathrm{GeV} / \mathrm{c}^{2}$, the Higgs boson decays predominantly into a bottom-antibottom quark pair and the process $q \bar{q} \rightarrow V H \rightarrow l l b \bar{b}$, where $V=W$ or $Z$, has the largest sensitivity because of the large multi-jet QCD background to the process $g g \rightarrow H \rightarrow b \bar{b}$. We look for a $b \bar{b}$ mass resonance in events containing a $\mathrm{W} / \mathrm{Z}$ boson, where the $W / Z$ decays leptonically into two or more jets. To suppress large backgrounds from $\mathrm{W} / \mathrm{Z}+$ jets 


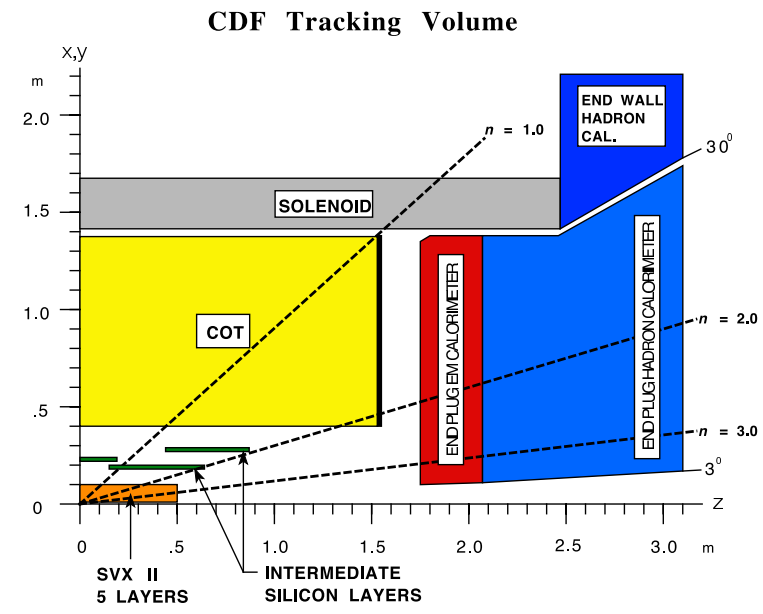

Figure 1: A schematic layout of the CDF tracking system.

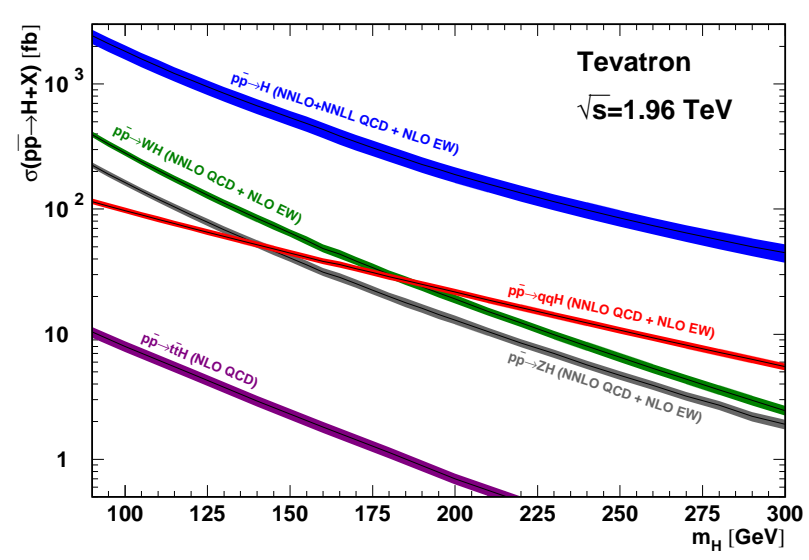

Figure 2: Cross sections for standard model Higgs boson production in $p \bar{p}$ collisions at $1.96 \mathrm{TeV}$ [4].

events, at least one jet must be b-tagged. Events are separated into final state categories based on the number of detected leptons, the number of jets and the quality of the b-tag(s) [5][6][7].

The dijet mass resolution for signal events at CDF is expected to be $10-15 \%$ of their mean reconstructed mass [8] and the presence of a signal would appear as a broad enhancement in the invariant mass distribution of jets. Figure 4 shows the dijet invariant mass distribution of simulated Higgs events with two charged leptons from a $Z$ decay in the final state, for three different values of the Higgs mass. The dijet mass provides the greatest discrimination between signal and background. However, to enhance sensitivity the dijet mass is combined with other kinematic information in multivariate analysis (MVA) to optimize the separation of the Higgs signal

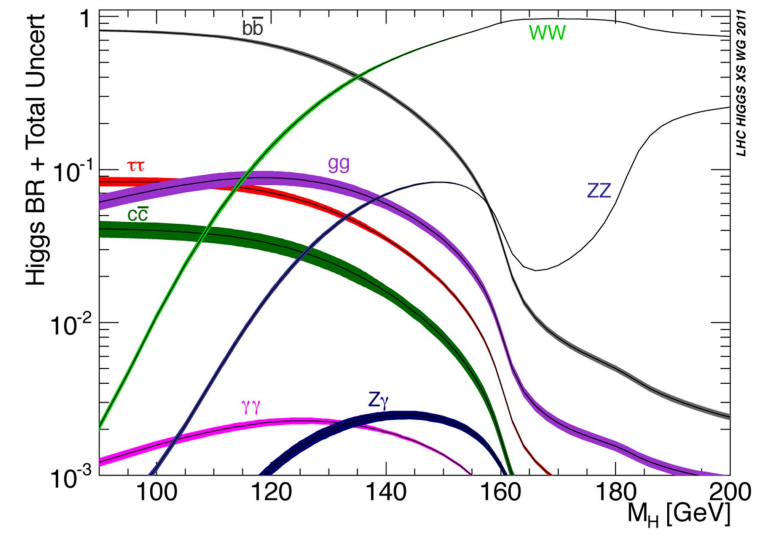

Figure 3: Branching ratios for the principal decays of standard model Higgs boson [4].

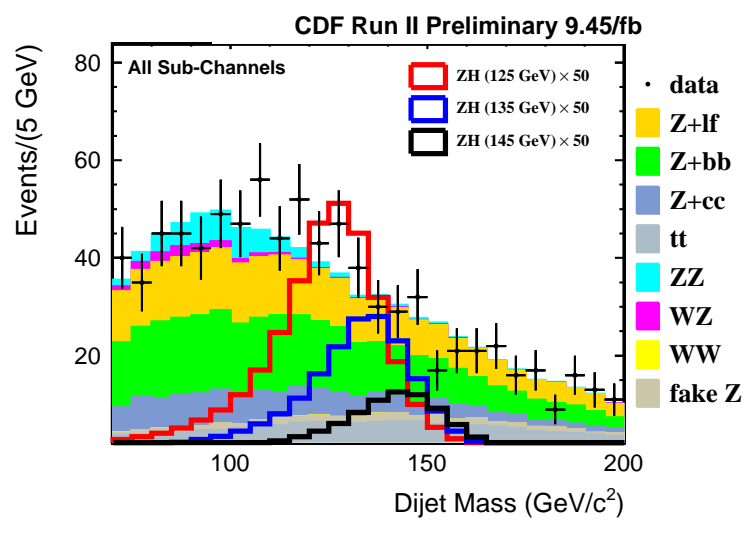

Figure 4: The dijet invariant mass distribution of simulated Higgs events with two charged leptons from a $Z$ decay and two jets in the final state, for three different values of the Higgs mass. The simulated backgrounds are shown as different shades and the data with circles.

from the backgrounds.

Above $130 \mathrm{GeV} / \mathrm{c}^{2}$, the $W W$ decay modes dominates and $g g \rightarrow H \rightarrow W W$ is the most sensitive process. For high mass signatures, we look for inclusive Higgs events, with the Higgs decaying into a WW pair. Events are separated into categories according to the number of jets and the number of leptons to take advantage of event kinematics that results from the different dominant signal and background processes associated with each final state. Each final state uses a customized MVA to separate the Higgs boson signal from the backgrounds.

The best overall sensitivity is obtained by combining all production and decay channels together. There are 71 mutually exclusive final states in the current CDF combined result, a complete list can be found in reference [9]. As a cross-check to establish the reliability of 
our analysis techniques, we use our analyses and combination machinery to measure $\mathrm{WZ} / \mathrm{ZZ}$ production in final states with charged leptons, neutrinos and heavy flavor jets and determine a $3.2 \sigma$ measurement of the cross section, in good agreement with the SM [1][10].

\section{Results}

All final states provide binned histograms of the final discriminant (MVA output) for data events, simulated signal events and each background process. The most recent high-order calculations of the SM Higgs boson production cross section and decay branching ratio are used to normalize the signal event yield for each individual channel, and the channels combined statistically. A combined likelihood function based on Poisson probabilities is constructed from the information contained in the individual bins of the expected and observed distributions of the final discriminant for each channel. Thus, the information in the background dominated bins is retained without diluting the power of the bins with the largest signal to background ratio. The number of selected events after subtraction of the expected background is shown in figure 5, as a function of $\log _{10}(s / b)$, where $s$ is the number of expected signal events and $b$ is the expected number of background events. The ratio $s / b$ is a measure of the discriminating power of the MVA bin containing the event. Also shown as a shaded histogram in figure 5 is the distribution of simulated signal events for a SM Higgs with mass of $125 \mathrm{GeV} / \mathrm{c}^{2}$.

The systematic uncertainties in the background and data models are treated as nuisance parameters, assuming a Gaussian probability distribution function for each. The likelihood function also depends on the values of these nuisance parameters. Using a Bayesian approach to determine either an upper limit or a best fit value for the SM Higgs production cross section, the nuisance parameters are integrated out to determine posterior probabilities. Details of the statistical treatment can be found in reference [9].

Assuming a signal is truly absent, the $95 \%$ credibility level limits on Higgs production are calculated, along with the significance of the excess in the data. Figure 6 shows the observed and expected limits for the individual analysis channels and the combined limits. Figure 7 shows the combined limit in more detail. The dashed line is the average limit from background-only pseudo experiments. The green and yellow bands contain the central $67 \%$ and $95 \%$ of the pseudo experiment results. The regions of Higgs boson masses excluded are $90<$ $m_{H}<97 \mathrm{GeV} / \mathrm{c}^{2}$ and $149<m_{H}<175 \mathrm{GeV} / \mathrm{c}^{2}$. The

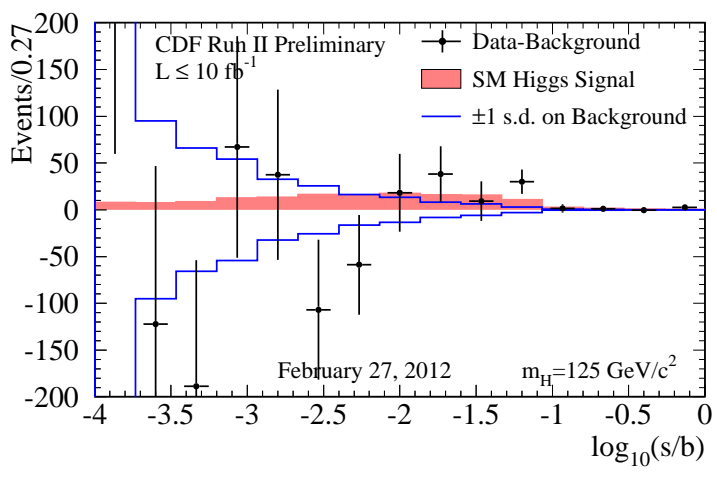

Figure 5: Combining all final states, the selected event yields after background subtraction are plotted in bins of $\log _{10}(s / b)$, a measure of the discriminating power of the MVA bin containing the event. Also shown as a shaded histogram is the distribution of simulated signal events for a SM Higgs with mass of $125 \mathrm{GeV} / \mathrm{c}^{2}$ and the uncertainty on the expected background as a solid line.

expected exclusion regions are $96<m_{H}<106 \mathrm{GeV} / \mathrm{c}^{2}$ and $154<m_{H}<176 \mathrm{GeV} / \mathrm{c}^{2}$. The excess observed in the data weakens the observed limits with respect to the expected limits. The observed $\mathrm{p}$-value as a function of Higgs mass, shown in figure 8 , exhibits a broad minimum and the maximum local significance corresponds to $2.6 \sigma$ at $m_{H}=120 \mathrm{GeV} / \mathrm{c}^{2}$. Correcting for the LookElsewhere Effect (LEE), which accounts for the possibility of a background fluctuation affecting the local pvalue anywhere in the search region, yields a global significance of $2.1 \sigma$. Fitting instead to the hypothesis of a standard model Higgs plus backgrounds, the best fit value for the production cross section is shown in figure 9 with its $1 \sigma$ uncertainty band.

By combining only the final states from the $q \bar{q} \rightarrow$ $V H \rightarrow l l b \bar{b}$ searches, a quasi model independent result can be obtained for the quantity $\left(\sigma_{W H}+\sigma_{Z H}\right) \times \operatorname{Br}(H \rightarrow$ $b \bar{b}$ ) in which only the SM ratio for $W H$ and $Z H$ production is assumed. The data for this restricted set of final states is shown in figure 10. The limits on SM Higgs production and corresponding p-values are in figure 11 and figure 12 . The p-value for the background only hypothesis at $m_{H}=125 \mathrm{GeV}^{2}$ is $2.5 \sigma$. The best fit value for $\left(\sigma_{W H}+\sigma_{Z H}\right) \times B r(H \rightarrow b \bar{b})$ as a function of the Higgs mass is shown in figure 13 with $1 \sigma$ and $2 \sigma$ uncertainty bands. Also shown is the $\mathrm{SM}$ prediction and its uncertainty as a thin line that decreases form left to right, and the corresponding shaded band . For a Higgs mass of $125 \mathrm{GeV} / \mathrm{c}^{2}$, we obtain $\left(\sigma_{W H}+\sigma_{Z H}\right) B r(H \rightarrow b \bar{b})=291 \pm_{113}^{118} \mathrm{fb}$. 


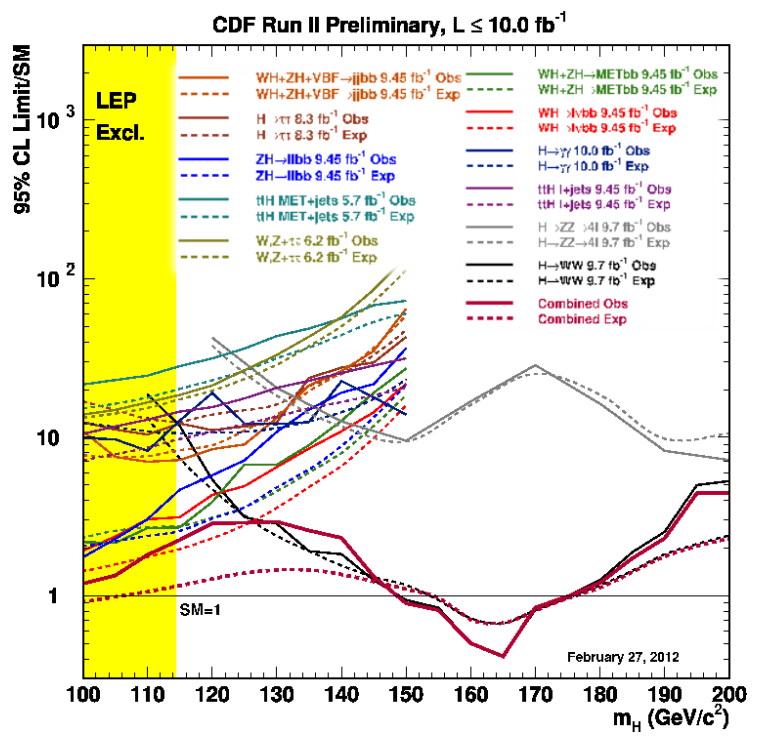

Figure 6: The expected (dashed line) and observed (solid line) limits for individual search channels are shown as a function of the Higgs mass. The combined limit is shown in thicker lines. In order of decreasing expected sensitivity at $m_{H}=125 \mathrm{GeV} / \mathrm{c}^{2}$, the channels are $W H \rightarrow l v b b, H \rightarrow W W, Z H \rightarrow l l b b, W H+Z H \rightarrow M E T b b, H \rightarrow \gamma \gamma$, $W H+Z H+V B F \rightarrow j j b b, t t H \rightarrow l+j e t s, H \rightarrow \tau \tau, W, Z+\tau \tau, H \rightarrow Z Z$, $t t H \rightarrow M E T+$ jets.

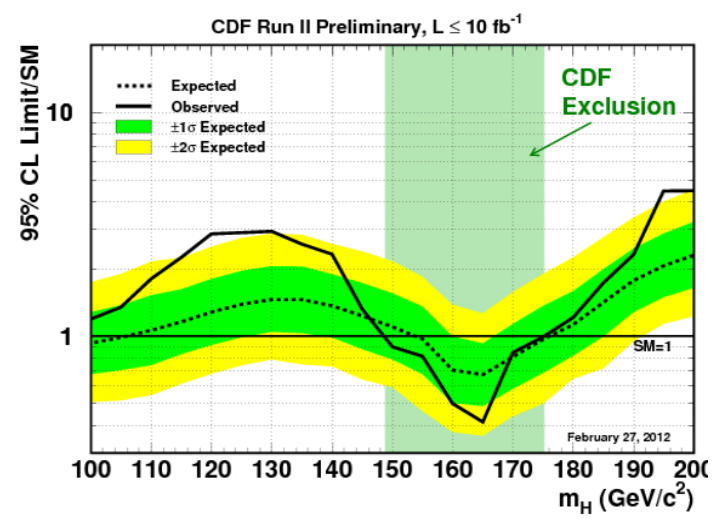

Figure 7: The expected (dashed line) and observed (solid line) limits for the standard model Higgs cross section as a function of the Higgs mass, obtained by combining all CDF SM Higgs searches. The shaded bands indicate the $1 \sigma$ and $2 \sigma$ uncertainties on the expected limit.

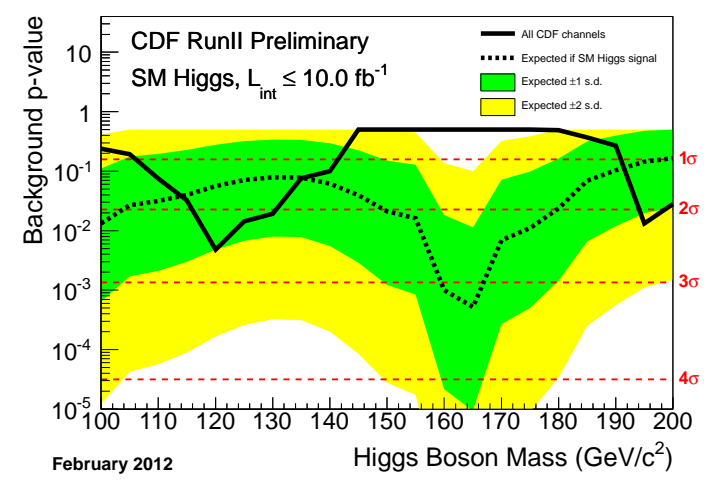

Figure 8: The probability for a fluctuation in the background to produce the observed number of excess events (background p-value) as a function of Higgs mass for the combination of all CDF SM Higgs searches.

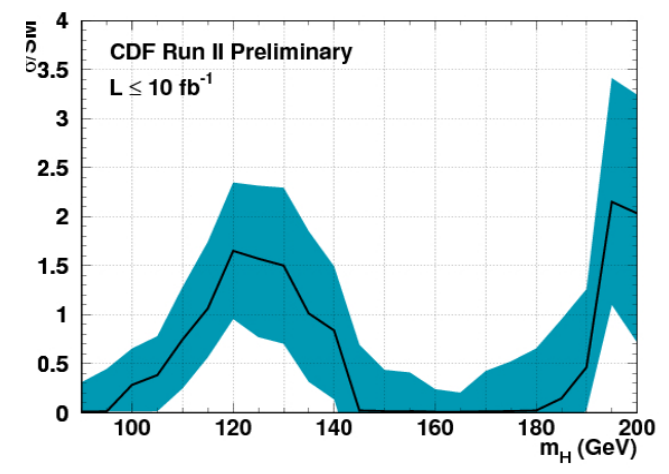

Figure 9: The best fit value of the SM Higgs production cross section as a function of the Higgs mass. The shaded band indicates the $1 \sigma$ uncertainty. 


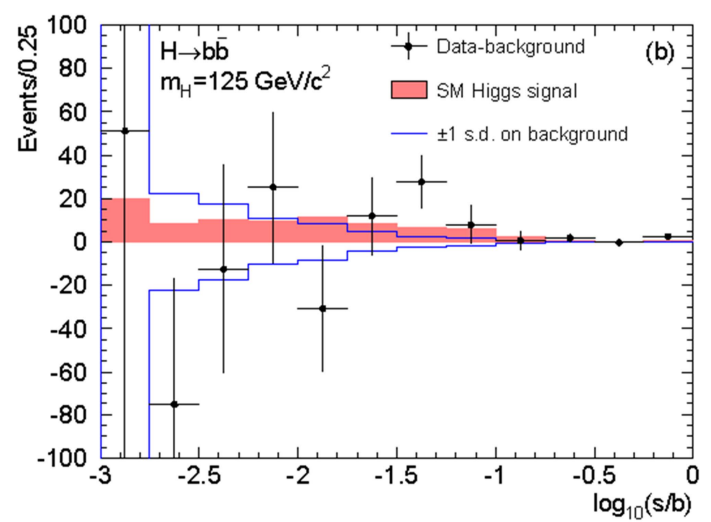

Figure 10: Combining all final states of associated production searches with $H \rightarrow b \bar{b}$, the selected event yields after background subtraction are shown in bins of $\log _{10}(s / b)$, a measure of the discriminating power of the MVA bin containing the event. Also shown as a shaded histogram is the distribution of simulated signal events for a SM Higgs with mass of $125 \mathrm{GeV} / \mathrm{c}^{2}$ and the uncertainty on the expected background as a solid line.

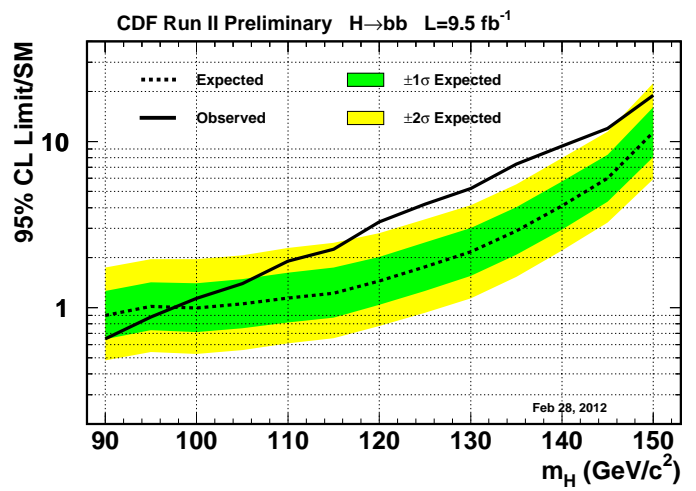

Figure 11: The expected (dashed line) and observed (solid line) limits for the standard model Higgs cross section as a function of the Higgs mass, considering only the searches for a Higgs boson produced together with a $\mathrm{W}$ or $\mathrm{Z}$ boson and decaying to a bottom-antibottom quark pair. The shaded bands indicate the $1 \sigma$ and $2 \sigma$ uncertainties on the expected limit.

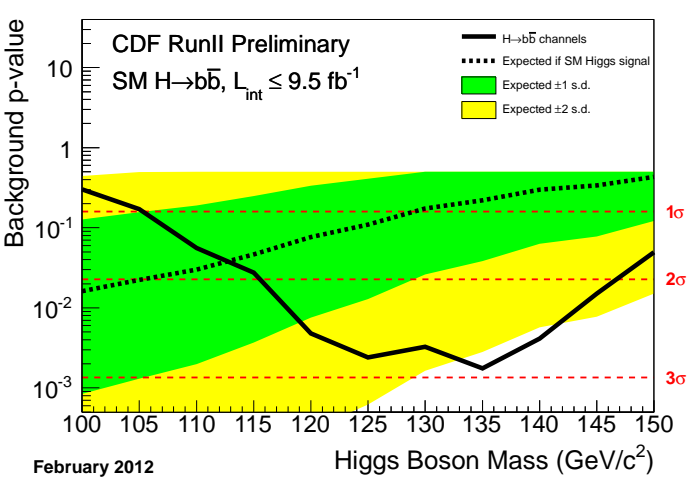

Figure 12: The background p-value as a function of Higgs mass for the combination of CDF searches for a Higgs boson produced together with a $\mathrm{W}$ or $\mathrm{Z}$ boson and decaying to a bottom-antibottom quark pair.

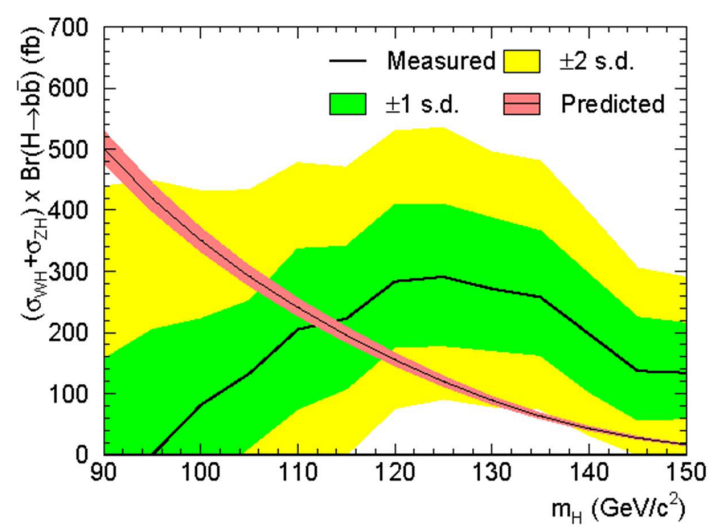

Figure 13: The thick line shows the best fit value of the quantity $\left(\sigma_{W H}+\sigma_{Z H}\right) \operatorname{Br}(H \rightarrow b \bar{b})$ as a function of the Higgs mass. The shaded bands indicate the $1 \sigma$ and $2 \sigma$ uncertainties. The SM prediction is shown as a thin line that decreases from left to right, and the surrounding shaded band shows the theoretical uncertainty. 


\section{Conclusions}

The most recent CDF direct Higgs search combination results have been presented. Using integrated luminosity of up to $10.0 \mathrm{fb}^{-1}$ and combining all final states, we observe an excess of events compatible with a standard model Higgs boson in the mass range $125 \mathrm{GeV} / \mathrm{c}^{2}$ and inconsistent with the background-only hypothesis at the level of $2.1 \sigma$. To access direct information about the Higgs boson's coupling to b-quarks, we combine the subset of search channels for which the Higgs is produced together with a $\mathrm{W}$ or $\mathrm{Z}$ boson, where the $\mathrm{W}$ or $\mathrm{Z}$ decays leptonically, and the Higgs decays to a bottomantibottom quark pair. An excess of events is observed, with a global significance of $2.5 \sigma$, and compatible with the standard model at the $2 \sigma$ level in the mass range $90-133 \mathrm{GeV} / \mathrm{c}^{2}$. For a Higgs mass of $125 \mathrm{GeV} / \mathrm{c}^{2}$, we obtain $\left(\sigma_{W H}+\sigma_{Z H}\right) \times B r(H \rightarrow b \bar{b})=291 \pm_{113}^{118} \mathrm{fb}$.

\section{References}

[1] T. Aaltonen, et al., Phys.Rev.Lett. 109 (2012) 111802.

[2] CMS Collaboration, "Search for the standard model Higgs boson produced in association with $\mathrm{W}$ or Z bosons, and decaying to bottom quarks for ICHEP 2012", CMS-PAS-HIG-12-019 (2012).

[3] CDF Collaboration, "The CDF II Detector Technical Design Report", FERMILAB-Pub-96/390-E (1996).

[4] J. Beringer, et al., Phys. Rev. D 86 (2012) 01001.

[5] T. Aaltonen, et al., Phys.Rev.Lett. 109 (2012) 111804.

[6] T. Aaltonen, et al., Phys.Rev.Lett. 109 (2012) 111805.

[7] T. Aaltonen, et al., Phys.Rev.Lett. 109 (2012) 111803.

[8] T. Aaltonen, et al., Phys. Rev. D 84 (2011) 071105.

[9] CDF Collaboration, "Combination of CDF's searches for the standard model Higgs boson with up to 10.0 1/fb of data", CDF Conference Note 10804 (2012).

[10] CDF Collaboration, "Measurement of WZ and ZZ production in final states with b-tagged jets", CDF Conference Note 10805 (2012). 